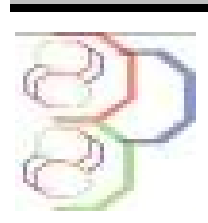

\title{
Mesure de la vitesse d'infiltration des eaux dans le sol : Cas des sols de la Vallée du Niari en République du Congo
}

\author{
Urbain Gampio MBILOU 1; Médard NGOUALA MABONZO* 2, Timothée MIYOUNA 1 \\ 1 Département de Géologie, Faculté des Sciences et Techniques ; Université Marien NGOUABI. BP : 69 Brazzaville, \\ République du Congo. \\ 2 Département de Géographie, Faculté des Lettres et Sciences Humaines ; Université Marien NGOUABI. BP : 69 \\ Brazzaville, République du Congo. \\ *Auteur correspondant, medngouala@yahoo.fr
}

Original submitted in on $10^{\text {th }}$ February 2016. Published online at www.m.elewa.org on $30^{\text {th }}$ April 2016 http://dx.doi.org/10.4314/iab.v100i1.1

\section{RESUME}

Objectifs : Au Congo, plusieurs projets agricoles sont en cours pour pallier à la dépendance étrangère sur les fruits, légumes et autres produits agricoles. Pour ceux, il est nécessaire d'opter pour une agriculture moderne et mécanisée. Pour éviter la pollution des nappes d'eau souterraine et des sols irrigués, les études de la vitesse d'infiltration des sols doivent être menées au préalable.

Méthodologie et résultats: Les mesures (profondeur d'infiltration, temps, vitesse) ont été effectuées directement sur le terrain. Le repérage du point exact de l'exécution de la mesure a été faite avec l'utilisation du GPS. Pour chacun de six points d'analyses, ont été réalisés trois mesures en même temps, en utilisant les trois filtres de double cylindre. La fréquence de mesure est de une minute pour les premiers 10 minutes

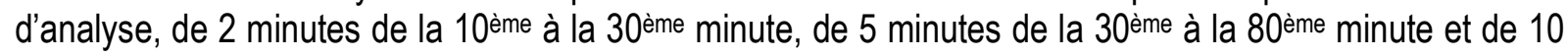

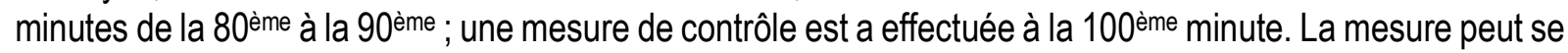
prolonger jusqu'à la 130 ème minute si la différence de vitesse entre les deux dernières mesures est supérieure à $10 \%$. La règle de mesure est graduée (de : $130-140 ; 190-200$ et $250-260$ ) puis associée aux filtres. Chaque filtre est gradué de bas en haut :0 à $10 \mathrm{~cm}$ (pour le filtre interne et externe), 0 à $5 \mathrm{~cm}$ doit être enfoncé dans le sol, 5 à $10 \mathrm{~cm}$ restent toujours rempli d'eau. Quand la hauteur d'eau descend à $5 \mathrm{~cm}$, on ajoute de l'eau pour la ramener à $10 \mathrm{~cm}$.

Conclusion : Les vitesses d'infiltration sont en rapport avec les différents horizons des sols : plus ou moins sablo argileux au sommet et argileux à la base. L'horizon argileux de la base ralentit considérablement l'infiltration et se comporte en véritable couche protectrice de la nappe d'eau souterraine. C'est donc une infiltration superficielle qui ne peut pas modifier la composition des eaux de l'aquifère. Ces sols sont alors favorables à une telle agriculture.

Mots clés : mesure, vitesse, infiltration, sols, pollutions, eau, Niari, Congo 


\section{Mbilou et al. J. Appl. Biosci. 2016 Mesure de la vitesse d'infiltration des eaux dans le sol : Cas des sols de}

la Vallée du Niari en République du Congo

Measuring the speed of the water infiltration into the soil: case of the soil of the Niary Valley in the Republic of Congo

Objectives: In Congo, several agricultural projects are underway to alleviate the dependence on foreign fruits, vegetables and other agricultural products. Therefore, it is necessary to opt for modern, mechanized agriculture. To prevent pollution of underground aquifers and irrigated soils, studies of soil infiltration rate must be carried out beforehand.

Methods and Results: The measurements (of depth, time and speed) were carried out directly in the field. Locating the exact point of execution of the measurement was made with using the GPS. For each of six points of analysis were carried out three measurements simultaneously, using the three double cylinder filters. The measurement frequency is one minute for the first 10 minutes of analysis, 2 minutes from the 10 th to the 30th minute, 5 minutes from the 30th to the 80th minute and 10 minutes from the 80th to the 90th; a control measurement is performed at a 100 th minute. The measurement can be prolonged until the 130 th minute if the speed difference between the last two measurements is greater than $10 \%$. The scale is graduated (: $130-140$; 190 - 200 and 250 - 260) and associated filters. Each filter is scaled from the bottom up: 0 to $10 \mathrm{~cm}$ (for the inner and outer filter) 0 to $5 \mathrm{~cm}$ must be driven into the ground; 5 to $10 \mathrm{~cm}$ are still filled with water. When the water level drops to $5 \mathrm{~cm}$, water is added to bring it to $10 \mathrm{~cm}$

Conclusion: The infiltration rates are related to the different soil horizons more or less sandy clay and clay at the top to the base. The clay horizon of base significantly slows infiltration and acts beneficially protective layer of the water table. This superficial infiltration cannot change the composition of the waters of the aquifer (underground water). These soils are so favourable for agriculture.

Keywords: measurement, speed, infiltration, soil, pollution, water, Niary, Congo

\section{INTRODUCTION}

Le Département du Niari est l'un des dix départements que compte la République du Congo. Situé au sud-ouest du pays, il figure parmi ceux qui ont une vocation agricole au Congo mais malheureusement mal exploité. Martin G (1970) a écrit: " la méconnaissance des potentialités et aptitudes culturales des sols ainsi que des techniques de cultures conservatrices, a constitué un lourd handicap pour le développement agricole de la vallée du Niari». Les ressources en eau sont utilisées en agriculture : i) dans de grandes exploitations agricoles modernes et privées (supérieures à $1 \mathrm{ha}$ ); ii) dans de petites exploitations privées (inférieures à $1 \mathrm{ha}$ ); iii) dans de petites exploitations paysannes (inférieures à 0.1 ha). Tous ces exploitants ne sont pas organisés en associations d'usagers. L'exploitation des ressources en eau n'est assujettie à aucune imposition ni tarification (AGRICONGO, 2003). Le potentiel des terres irrigables du Congo est de 340000 ha. La régularité des précipitations dans la majeure partie du pays a favorisé le développement des cultures pluviales au détriment des cultures irriguées. La principale technique d'irrigation utilisée en maîtrise totale est l'irrigation de surface et les principales sources de captage des eaux pour l'irrigation sont les rivières et les plans d'eau de surface (FAO, 2005).En ce qui concerne le choix des terres à mettre en valeur, les critères utilisés ne se sont pas révélés toujours très heureux (Martin, 1970). L'inventaire des sols entrepris par (Brugère, 1953) a révélé de grandes variations texturales. (Henin, 1956) en calculant l'indice d'instabilité structurale des sols du Niari a obtenu des valeurs inferieures à 1. Leur texture est variable et en relation avec les différentes roches sous-jacentes : sablo-argileuse sur quartzites, argilolimoneuse sur schistes fins, le plus généralement argileuse-sableuse à sables plus ou moins grossiers. Le ruissellement, en raison de l'importante couverture forestière, est moins fort que celui auquel on pourrait s'attendre en raison des fortes pentes et de la faible perméabilité. (Hiez, 1965). En étudiant les conditions de mécanisation de ces sols (Cavala, 1960) a été amené à concevoir des façons culturales plus appropriées par un travail du sol sans retournement avec des engins à disques crénelés type Rome-Plow et à les réduire tout en conservant des rendements au moins égaux à ceux obtenus avec les façons classiques. Une agriculture moderne 
exige des moyens mécaniques, de l'eau pour l'irrigation, l'utilisation des engrais et de grandes étendues pour ne citer que ceux là. Dans le but de préserver les nappes d'eau souterraines et les cours d'eau contre des éventuelles pollutions, et aussi de connaitre l'état des sols des domaines réservés à la mécanisation agricole ; les mesures de la capacité d'infiltration des sols paraissent plus que nécessaires : d’où l’objectif de notre présente étude.

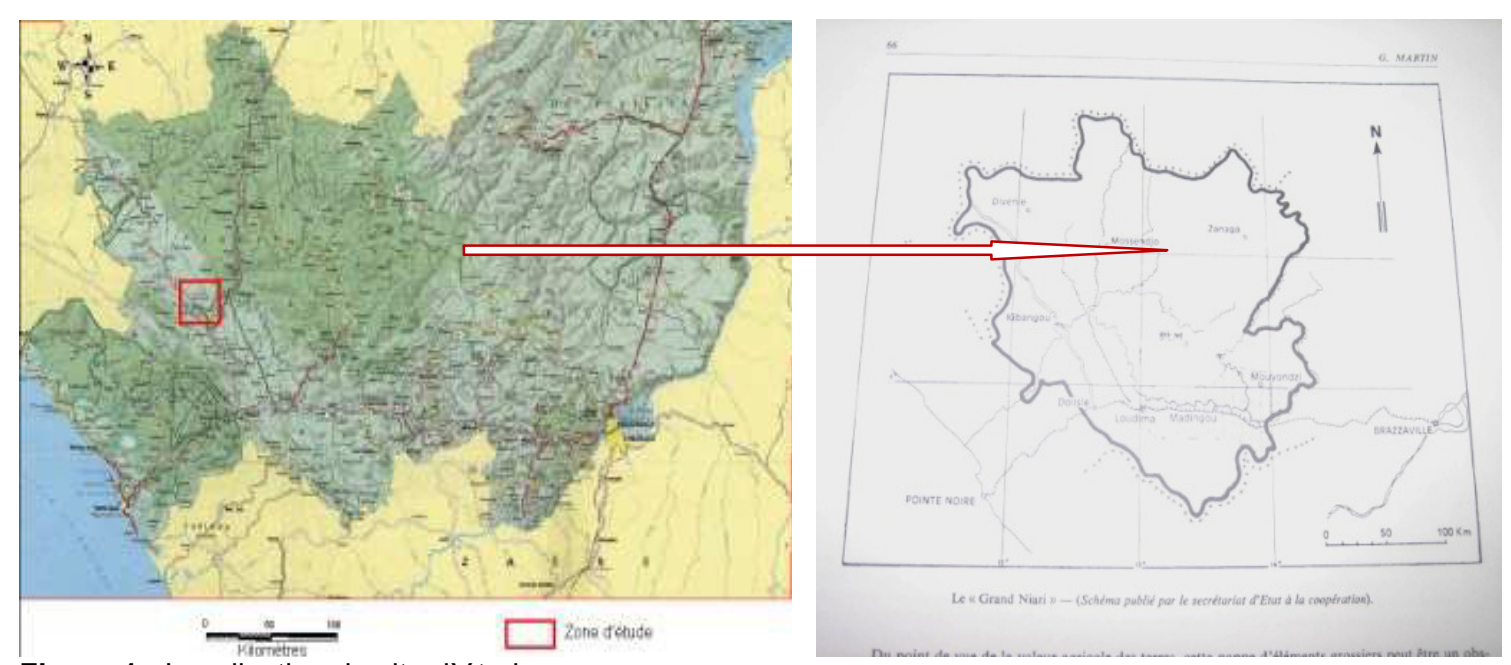

Figure 1 : Localisation du site d’étude.

Notre site d'étude se trouve dans la vallée du Niari. Cette vallée s'allonge et s'élargit d'Est en Ouest. C'est une plaine d'érosion entaillée dans des roches schistocalcaires, trouée de dolines et d'autres dépressions fermées. Cette plaine débouche à l'ouest dans une longue et ample dépression sud-est - nord-ouest drainée par le Niari et la Nyanga. Elle comporte de vastes étendues planes où les phénomènes karstiques ont une grande extension ; les plus spectaculaires sont des groupes serrés de pitons coniques, tels que les « Monts de la Lune ", près de la boucle du Niari. Dans la partie occidentale se succèdent des chaînons coiffés de couches gréseuses qui dessinent des alignements séparés par des dépressions humides. Ils atteignent 750 $\mathrm{m}$ près de la frontière avec la République Démocratique du Congo (RDC). Le collecteur le plus important dans ce département est le fleuve Niari, qui n'est autre que le cours moyen du fleuve Kouilou et encore appelé Ndouo dans son cours supérieur. Son module interannuel est de $930 \mathrm{~m}^{3} / \mathrm{s}$ dans son cours inférieur à Sounda près de Kakamoéka. Les sous-bassins importants sont ceux de la Louessé $\left(15630 \mathrm{~km}^{2}\right.$ et $302 \mathrm{~m}^{3} / \mathrm{s}$ à Makabana) et la Bouenza (4 $920 \mathrm{~km}^{2}$ et $112 \mathrm{~m}^{3} / \mathrm{s}$ à Miambou). Depuis sa source, sur les Plateaux Batéké jusque dans le synclinoruim schisto-calcaire, le fleuve Niari traverse différentes formations géologiques (sables Batéké, argilites et grès bouénziens, tillite supérieure, calcaires et marnes du Niari).Par ailleurs, ses principaux affluents du cours moyen apportent d'autres matériaux, soit de la zone granitique (pour la Bouénza), soit du Schistogréseux (pour la Nkenké et la Loudima). Il en résulte que les alluvions du Niari et de ses affluents peuvent être constituées de matériaux très hétérogènes arrachés aux différentes roches situées en amont. Présentes dans les régions tropicales humides sur environ vingt pour cent de la surface des continents, les couvertures ferralitiques sont marquées par une forte expression des constituants ferrifères (hématite $\mathrm{Fe}_{2} \mathrm{O}_{3}$; Goethite $\mathrm{FeO}, \mathrm{OH}$ ) résultant de l'altération météorique des roches diverses. Ces formations superficielles zonales se caractérisent essentiellement par leur grande épaisseur (fréquemment plus de 10 mètres), par leur couleur vive [de $5 R$ (rouge) à 10 YR (jaune-rouge) selon le code Munsell], et par l'accumulation de produits résiduels constitués d'hydroxydes métalliques et d'argiles essentiellement monosiallitiques. Ces produits résiduels de l'altération et de la pédogénèse ferralitiques, qui comprennent parfois des cuirasses latéritiques, ferrugineuses, bauxitiques et manganésifères, ont été reconnus et étudiés depuis le début du XXème siècle par de nombreux auteurs. Des transferts de matières postérieurs à l'altération des minéraux primaires aboutissent à diverses accumulations absolues qui se succèdent dans l'infrasol. Depuis les latérites jusqu'aux horizons meubles, la porosité tissurale 
héritée des roches mères est relayée et/ou complétée par une forte porosité intra et intercristalline (lors de la dissolution des minéraux primaires), puis recoupée par une porosité tubulaire d'origine biologique. Toutes ces porosités peuvent être le siégé de dépôts cutaniques argilo-métalliques (ferrugineux ou alumineux), ou de dépôts ultimes de micro agrégats biologiques (MULLER D. et al., 1981; BOULANGE, 1984), qui résultent de transferts de matière provenant des couches susjacentes. Il existe dans la Vallée du Niari une gamme de sols dont les potentialités et les aptitudes sont très diverses. Le profil des sols du Niari présente trois espaces: supérieur, moyen et inferieur. Le niveau supérieur présente généralement la morphologie suivante (ORSTOM Congo 1965, Pédologie n 731) :
$0-2 \mathrm{~cm}$ : humifère noir (10 YR 2/1), argileux, grumeleux fin, avec un chevelu racinaire fin dense, et une porosité élevée.

$2-30 \mathrm{~cm}$ : humifère homogène, brun sombre (10YR 2/2) argileux, structure nuciforme à polyédrique fine de cohésion moyenne.

30- $50 \mathrm{~cm}$ : horizon argileux d'aspect bigarré par suite d'une pénétration humifère hétérogène essentiellement sur les faces des agrégats polyédriques moyens à grossiers, qui sont colorés en brun (7,5 YR 5), tandis que la masse terreuse est jaune brunâtre (10 YR 6/6). En dessous la pénétration humifère devient peu visible, la structure est de type polyédrique moyen de $50 \mathrm{~cm}$, mais moins cohérente et se résolvant en une agrégation très fine de microgrumeleuse, de couleur jaune ocre (10 YR 6/6). Ce matériau présente alors une texture argileuse, assez constante lorsque s'enfonce dans le profil.

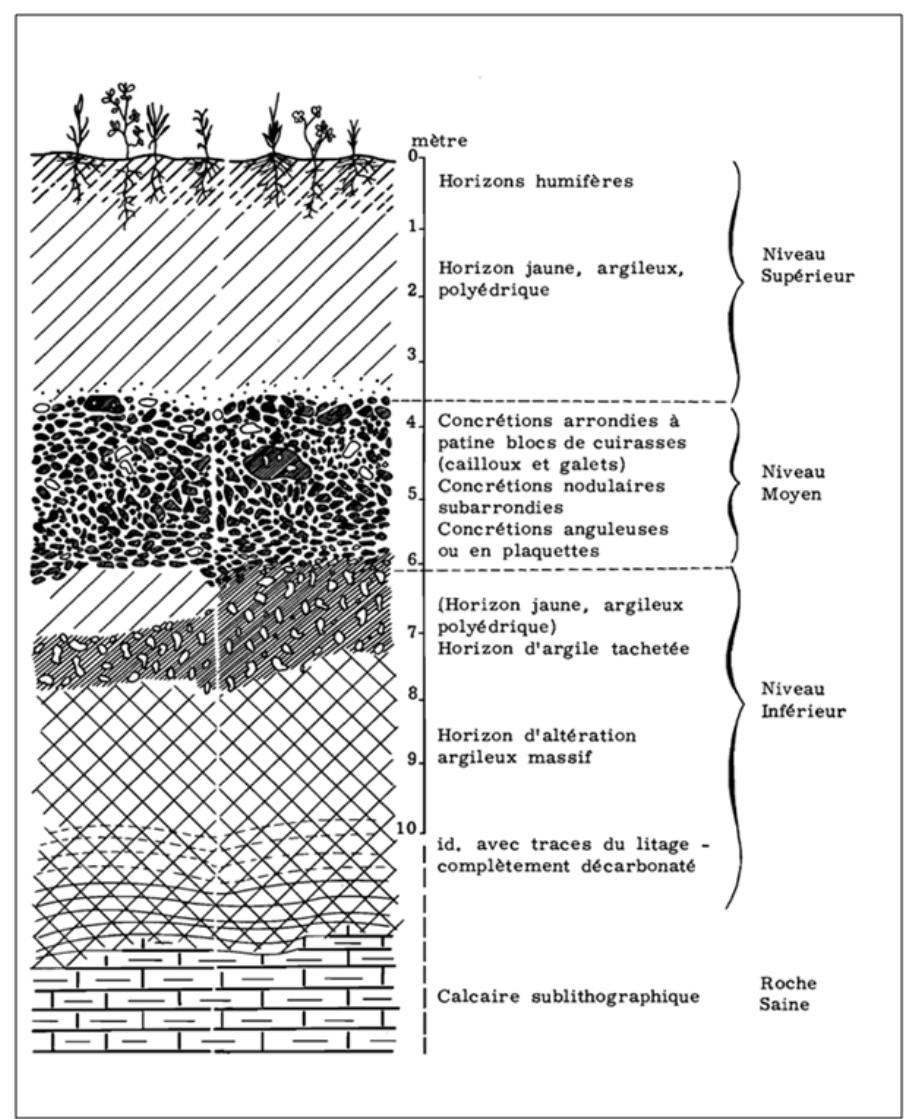

Figure 2 : Profil des sols complexes sur schisto calcaire (ORSTOM Brazzaville 1965, Pédologie $n^{\circ} 731$ )

Du point de vue de la valeur agricole des terres, cette nappe d'éléments grossiers peut être un obstacle au développement racinaire des plantes cultivées lorsqu'elle est située à une faible profondeur utile (sols érodés).
Lorsque les sols ont une profondeur utile suffisante, leurs caractéristiques fondamentales sont surtout liées à la nature des matériaux du niveau supérieur meuble. Les caractéristiques physico-chimiques des matériaux de ces 


\section{Mbilou et al. J. Appl. Biosci. 2016 Mesure de la vitesse d'infiltration des eaux dans le sol : Cas des sols de la Vallée du Niari en République du Congo}

sols montrent qu'ils ont subi une altération de type ferralitique, mais généralement pas très poussée, et l'on peut classer la plupart des sols de la Vallée du Niari dans le groupe des sols faiblement ferralitiques Toutefois certains sols formés sur matériaux d'apport (alluviaux ou colluviaux) relativement récents, présentent un degré d'évolution moins poussé et se classent donc à la limite inférieure de la sous-classe des sols ferrallitiques. Du fait de l'altération ferralitique, ces sols ont généralement une réserve minérale très limitée et les éléments assimilables surtout concentrés dans les horizons humifères sont également peu abondants. II en résulte que les taux de saturation du complexe absorbant sont généralement très bas (moins de $20 \%$ ) et la réaction des sols est acide (pH voisin de 5 ou parfois moins). La fraction argileuse de ces sols est surtout constituée de kaolinite et de goethite, avec parfois de l'illite ; c'est dire que la capacité d'échange de la fraction minérale des sols n'est jamais très importante ( 6 à $10 \mathrm{~m} / 100 \mathrm{~g}$ d'argile) et le pouvoir de rétention pour l'eau limité. La matière organique de ces sols, essentiellement issue de la décomposition du système racinaire de la savane, confère cependant aux horizons supérieurs une capacité d'échange cationique (180 $\mathrm{m} / 100 \mathrm{~g}$ de matière organique) et des possibilités de rétention pour l'eau nettement plus élevées. Elle joue aussi un rôle important en rendant plus fine la structure des sols, en augmentant la stabilité des agrégats terreux, ainsi que la perméabilité des horizons supérieurs. Cette matière organique des sols joue donc un rôle primordial en ce qui concerne les propriétés physiques et chimiques de ces sols. Du point de vue biologique, les sols sous végétation naturelle présentent une activité biologique globale relativement élevée par rapport aux autres sols congolais, avec en particulier un cycle du carbone rapide. Par contre le cycle de l'azote paraît moins actif : La fixation d'azote atmosphérique (Beijerinckia) est moyenne, mais la minéralisation des matières azotées du sol et la nitrification paraissent se faire assez mal. Si nous classons les sols de la Vallée du Niari d'après leur potentiel de fertilité (Martin, 1970), nous trouvons au premier lieu les sols alluviaux. II s'agit de sols d'origine ferralitique et remaniés par alluvionnement. Ils n'occupent que des surfaces réduites et dispersées surtout le long du Niari et de certains affluents. Leur potentiel chimique est relativement bon, ainsi que les qualités physiques, et justifieraient des travaux d'aménagement (drainage, irrigation). A l'heure actuelle, ils sont fort peu utilisés en culture industrielle, mais servent à des cultures vivrières manuelles avec une rotation culture-jachère assez accélérée. En second lieu figurent les sols argileux de plateau, (plateau de la Vallée du Niari et plateaux de
Mouyondzi). Ce sont les sols qui, du fait de l'absence de forte pente, se prêtent le mieux à une exploitation agricole de type mécanisé et, de fait, ils ont été largement cultivés entre Loudima et Madingou (culture mécanisée) et sur les plateaux de Mouyondzi (culture manuelle). Ces sols sont généralement, suffisamment profonds, sauf parfois en bordure des plateaux (affleurement de l'horizon gravillonnaire), et, malgré une texture lourde, ils ont une structure assez fine pour permettre de nombreuses cultures y compris l'arachide. Leur potentiel chimique est par contre faible. Une mention particulière doit être faite pour les sols colluviaux de bas de pente que l'on trouve dans et en bordure de la zone des plateaux de la Vallée du Niari. Ils bénéficient d'une alimentation en eau supérieure et de propriétés chimiques plus intéressantes. Étant donné l'exiguiité et la pente des parcelles, seules des cultures manuelles sont possibles. Une intensification des cultures vivrières sur ces terres peut être envisagée. Les sols argilo-sableux à sablo-argileux de la plaine de piedmont présentent des potentialités chimiques médiocres, analogues à celles des sols argileux ; de plus, leurs propriétés physiques sont moins favorables, du fait de leur structure moins fine et moins stable et d'une tendance à la compaction des horizons profonds (pseudogley). La présence fréquente de nombreuses dépressions marécageuses et de sols dont la profondeur utile est réduite (affleurement d'horizon gravillonnaire), les rend moins favorables pour la grande culture que les sols argileux des plateaux. De plus, l'expérience a montré que l'exploitation de ces terres était encore plus délicate que pour les sols argileux de plateau. Toutefois une exploitation semi-intensive agro-pastorale peut y être envisagée.

En quatrième position on trouve les sols colluviaux situés au pied des reliefs calcaires ou dolomitiques. Ces sols présentent une richesse minérale intéressante, quoique souvent déséquilibrée. Lorsque ces sols ne sont pas trop pierreux, ni hydromorphes, ils fournissent de petites parcelles qui donnent de beaux produits. Les autres catégories de sols que l'on observe dans la Vallée du Niari présentent des potentialités agricoles nettement moins intéressantes. Les roches des niveaux schistecalcaires contenant des calcaires, calcaires dolomitiques et dolomies, massifs et bien cristallisés $\left(\mathrm{SC}_{l^{a}}, \mathrm{SC}_{l^{\mathrm{c}}}\right.$, et une grande partie du $\mathrm{SC}_{\text {III) }}$ se désagrègent et s'altèrent relativement lentement. Les roches des autres niveaux du schiste-calcaire, généralement riches en minéraux argileux (SC, ${ }^{b}$ et $\left.\mathrm{SC}_{\text {III }}\right)$ sont par contre à l'origine de sols bien développés. Enfin les sols issus de matériaux originels provenant du schiste-gréseux paraissent résulter de la décomposition de grès à grains fins, généralement 
feldspathiques et d'argilites de l'étage inférieur de la série de la M'Pioka. En bordure du Niari et de ses principaux affluents, les sols des terrasses sont formés à partir de matériaux alluviaux d'origines très diverses en fonction de l'hétérogénéité pétrographique des bassins versants. Bien que la plupart des sols soient issus de matériaux provenant de calcaire et de dolomie, on observe dans le Niari des sols extrêmement argileux et des sols sableux, avec de nombreux intermédiaires. En fait les sols ne paraissent pas provenir exclusivement et simplement de l'altération de roches locales, mais sont souvent issus d'un mélange de matériaux d'origines diverses qui ont

\section{MATERIELS ET METHODE}

Les matériels utilisés sont: l'infiltromètre à double anneau (photo 1) qui est un instrument simple, utilisé pour déterminer le taux d'infiltration d'eau dans le sol. Le taux d'infiltration correspond à la quantité d'eau, par unité

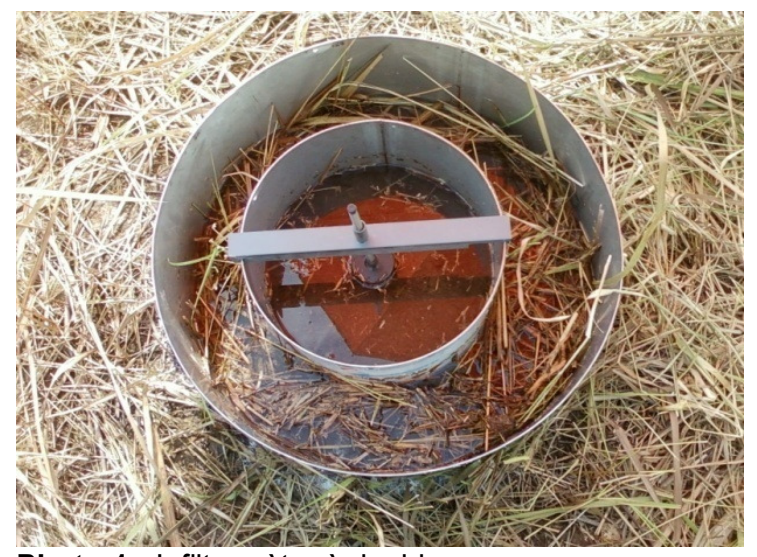

Photo 1 : Infiltromètre à double anneau

Légende : une règle de mesure avec flotteur (photo 2),

Les mesures ont été effectuées directement sur le terrain. Pour chacun de six unités cartographiques, a été évaluée la vitesse d'infiltration du sol. Le repérage du point exact de l'exécution de la mesure a été faite avec l'utilisation du GPS. Pour chacun de six points d'analyses, ont été réalisés trois mesures en même temps, en utilisant les trois filtres de double cylindre. Pour chaque mesure une fiche de campagne est à remplir (voir la fiche $n^{\circ} 1$ à la fin). La fréquence de mesure est de une minute pour les premiers 10 minutes d'analyse, de 2 minutes de la 10 ème

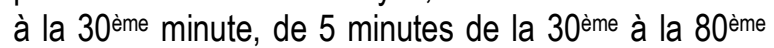

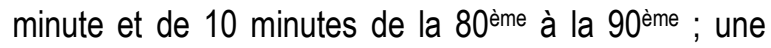
mesure de contrôle est a effectuée à la 100ème minute. La subi des remaniements et différents cycles de pédogenèses. Cette histoire pédogénétique complexe se traduit généralement dans les sols par le grand développement des profils et par la présence d'une nappe d'éléments grossiers dans laquelle sont rassemblés des matériaux ferrallitiques (concrétions, blocs de cuirasses, etc.) et des matériaux résiduels (cherts, galets ou débris de roches diverses, etc.). Cette nappe d'éléments grossiers est généralement surmontée d'un niveau meuble plus ou moins épais, mais remarquable par son homogénéité.

de surface et unité de temps qui pénètre dans le sol. $\mathrm{Ce}$ taux peut être calculé sur la base des résultats de mesures et/ou sur la base de la loi "Loi de Darcy". (Bouwer H., 1986).

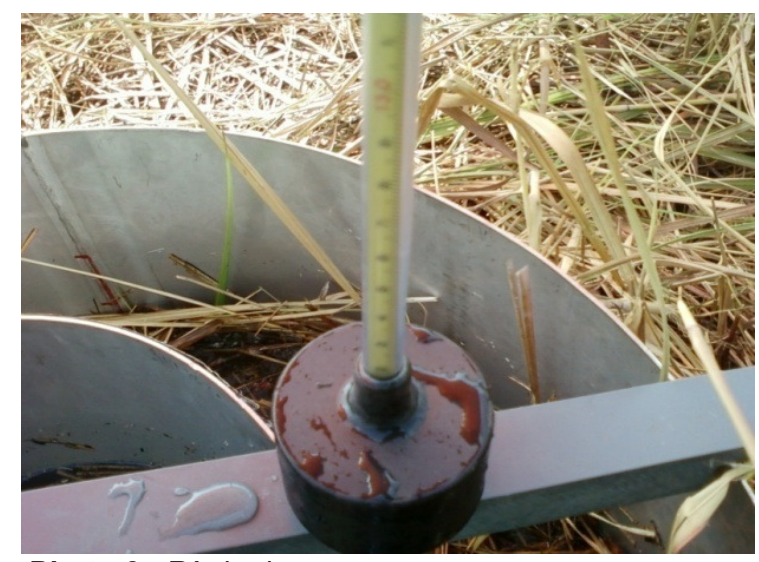

Photo 2 : Règle de mesure

la carte de la zone de projet, le GPS.

mesure peut se prolonger jusqu'à 120 ème ou 130ème minute si la différence de la vitesse (capacité) d'infiltration entre les deux dernières mesures n'est pas inférieur à $10 \%$. La règle de mesure est graduée (de : 130 - 140 ; $190-200$ et $250-260$ ) et est associée aux filtres.

Chaque filtre est gradué de bas en haut:

- 0 à $10 \mathrm{~cm}$ (pour le filtre interne et externe),

- 0 à $5 \mathrm{~cm}$ doit être enfoncé dans le sol,

- 5 à $10 \mathrm{~cm}$ doit être toujours rempli d'eau.

A tout moment que la hauteur d'eau descend jusqu'à 5 $\mathrm{cm}$, on doit obligatoirement ajouter de l'eau et ramener le niveau à $10 \mathrm{~cm}$. 


\section{Mbilou et al. J. Appl. Biosci. 2016 Mesure de la vitesse d'infiltration des eaux dans le sol : Cas des sols de la Vallée du Niari en République du Congo}

\section{RESULTATS}

Une feuille de protocole de mesure est rempli au fur et à mesure que l'expérience s'effectue directement sur le terrain ainsi que les calculs est présentée comme exemplaire au tableau $n^{\circ} 1$ ci-dessous et le reste des

résultats obtenus sont traduits sous forme de graphique du $n^{\circ} 1$ au $n^{\circ} 6$. Ils expriment les courbes d’évolution de la vitesse ( $G$, en $\mathrm{cm} / \mathrm{min}$ ) en fonction de la profondeur ( $F$, en $\mathrm{cm})$, ceci pour chaque point étudié.

Tableau 1 : Protocole de mesure de la vitesse d'infiltration des eaux dans le sol (cas du point n०1).

\begin{tabular}{|c|c|c|c|c|c|c|c|}
\hline \multicolumn{2}{|l|}{ Site } & \multicolumn{2}{|c|}{ Vallée du Niari } & \multicolumn{4}{|c|}{ Coordonnées géographique : S $03^{\circ} 29^{\prime} 40.6^{\prime \prime}$ et E $12^{\circ} 24^{\prime} 37.5^{\prime \prime}$} \\
\hline \multicolumn{4}{|c|}{ MBILOU Urbain G. } & & & & \\
\hline \multicolumn{8}{|c|}{$\begin{array}{ll}\text { Règle de mesure } & \mathrm{M} \mathrm{130-140}\end{array}$} \\
\hline$A$ & & 3 & C & $\mathrm{D}$ & $E$ & $\mathrm{~F}$ & G \\
\hline \multirow{2}{*}{$\begin{array}{l}\text { Heure } \\
\text { (hh, mm, } \\
\text { ss) }\end{array}$} & \multicolumn{2}{|c|}{ Niveau eau (cm) } & \multirow{2}{*}{$\begin{array}{l}\text { Temps } \\
\text { cumulatifs } \\
\text { (min) }\end{array}$} & \multirow{2}{*}{$\begin{array}{l}\text { Intervalles } \\
\text { du temps } \\
\text { (min) }\end{array}$} & \multirow{2}{*}{$\begin{array}{l}\text { Infiltration } \\
(\mathrm{cm})\end{array}$} & \multirow{2}{*}{$\begin{array}{l}\text { Infiltration } \\
\text { cumulatif }(\mathrm{cm})\end{array}$} & \multirow{2}{*}{$\begin{array}{l}\text { Capacité } \\
\text { (vitesse) } \\
\text { d’infiltration } \\
\mathrm{cm} / \mathrm{min}\end{array}$} \\
\hline & $\begin{array}{l}\text { Avant } \\
\text { remplissage }\end{array}$ & $\begin{array}{l}\text { Après } \\
\text { remplissage }\end{array}$ & & & & & \\
\hline \multirow[t]{31}{*}{$13 \mathrm{~h} 36$} & & 134,4 & 0 & start & start & start & start \\
\hline & 134,9 & & 1 & 1 & 0,5 & 0,5 & 0,5 \\
\hline & 135,2 & & 2 & 1 & 0,3 & 0,8 & 0,3 \\
\hline & 135,6 & & 3 & 1 & 0,4 & 1,2 & 0,4 \\
\hline & 136 & & 4 & 1 & 0,4 & 1,6 & 0,4 \\
\hline & 136,3 & & 5 & 1 & 0,3 & 1,9 & 0,3 \\
\hline & 136,6 & & 6 & 1 & 0,3 & 2,2 & 0,3 \\
\hline & 136,8 & & 7 & 1 & 0,2 & 2,4 & 0,2 \\
\hline & 136,9 & & 8 & 1 & 0,1 & 2,5 & 0,1 \\
\hline & 137,1 & & 9 & 1 & 0,2 & 2,7 & 0,2 \\
\hline & 137,2 & & 10 & 1 & 0,1 & 2,8 & 0,1 \\
\hline & 137,7 & & 12 & 2 & 0,5 & 3,3 & 0,25 \\
\hline & 138 & & 14 & 2 & 0,3 & 3,6 & 0,15 \\
\hline & 138 & & 16 & 2 & 0 & 3,6 & 0 \\
\hline & 138,1 & & 18 & 2 & 0,1 & 3,7 & 0,05 \\
\hline & 138,4 & & 20 & 2 & 0,3 & 4 & 0,15 \\
\hline & 138,8 & & 22 & 2 & 0,4 & 4,4 & 0,2 \\
\hline & 138,9 & & 24 & 2 & 0,1 & 4,5 & 0,05 \\
\hline & 139 & 134,3 & 26 & 2 & 0,1 & 4,6 & 0,05 \\
\hline & 134,5 & & 28 & 2 & 0,2 & 4,8 & 0,1 \\
\hline & 135 & & 30 & 2 & 0,5 & 5,3 & 0,25 \\
\hline & 136 & & 35 & 5 & 1 & 6,3 & 0,2 \\
\hline & 137 & & 40 & 5 & 1 & 7,3 & 0,2 \\
\hline & 137,9 & & 45 & 5 & 0,9 & 8,2 & 0,18 \\
\hline & 138,6 & 133,5 & 50 & 5 & 0,7 & 8,9 & 0,14 \\
\hline & 134,4 & & 55 & 5 & 0,9 & 9,8 & 0,18 \\
\hline & 135,4 & & 60 & 5 & 1 & 10,8 & 0,2 \\
\hline & 136,3 & & 65 & 5 & 0,9 & 11,7 & 0,18 \\
\hline & 137,2 & & 70 & 5 & 0,9 & 12,6 & 0,18 \\
\hline & 138,1 & & 75 & 5 & 0,9 & 13,5 & 0,18 \\
\hline & 138,7 & 134,6 & 80 & 5 & 0,6 & 14,1 & 0,12 \\
\hline 15h07 & 136,3 & & 90 & 10 & 1,7 & 15,8 & 0,17 \\
\hline
\end{tabular}


Mbilou et al. J. Appl. Biosci. 2016 Mesure de la vitesse d'infiltration des eaux dans le sol : Cas des sols de la Vallée du Niari en République du Congo

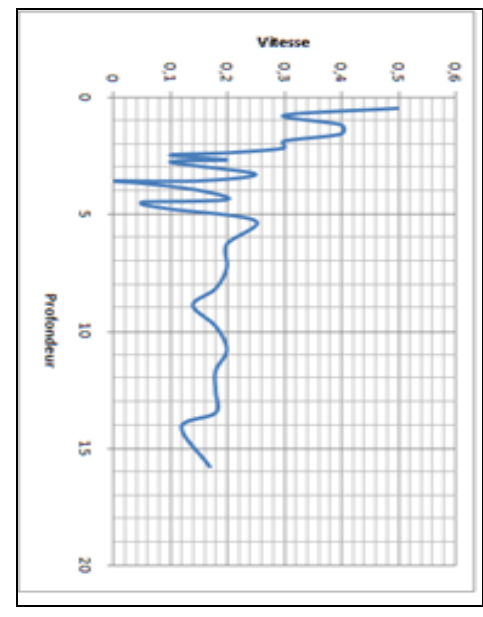

Figure 3 : vitesse $(\mathrm{cm} / \mathrm{min})$ d'infiltration au point $n^{\circ} 1$

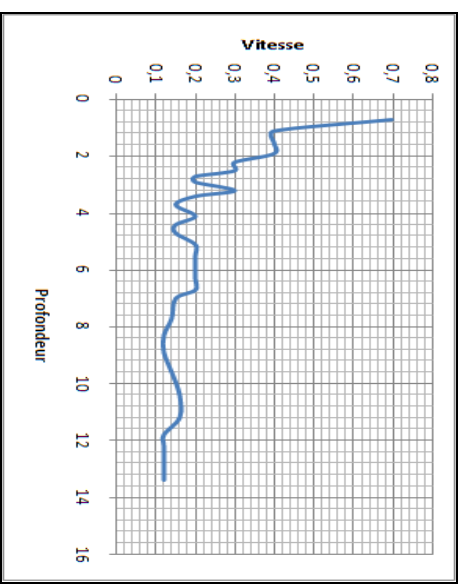

Figure 5 : vitesse $(\mathrm{cm} / \mathrm{min})$ d'infiltration au point $n^{\circ} 3$

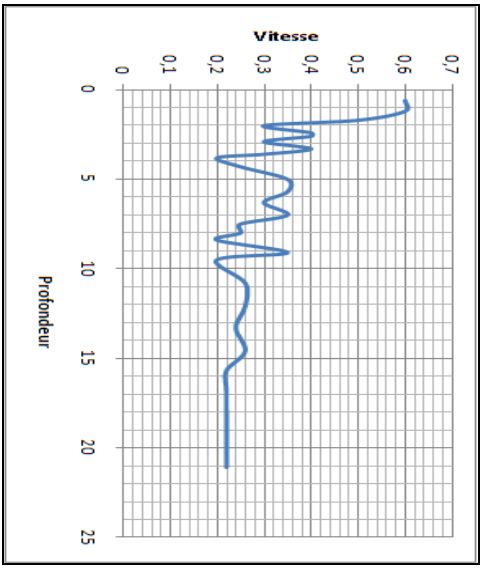

Figure 7: vitesse $(\mathrm{cm} / \mathrm{min})$ d'infiltration au point $n^{\circ} 5$

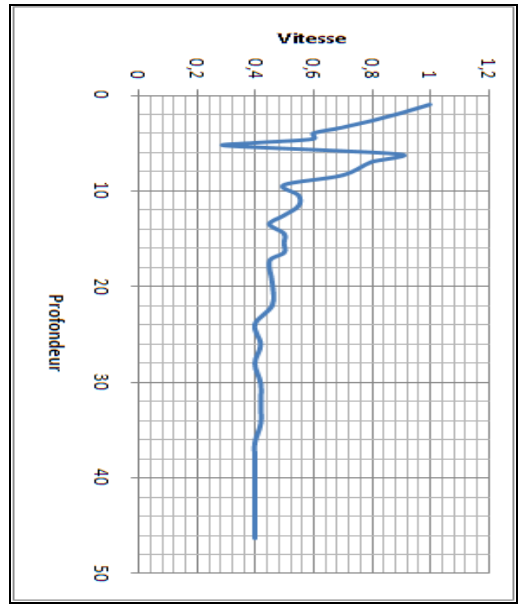

Figure 4 : vitesse $(\mathrm{cm} / \mathrm{min})$ d'infiltration au point $n^{\circ} 2$

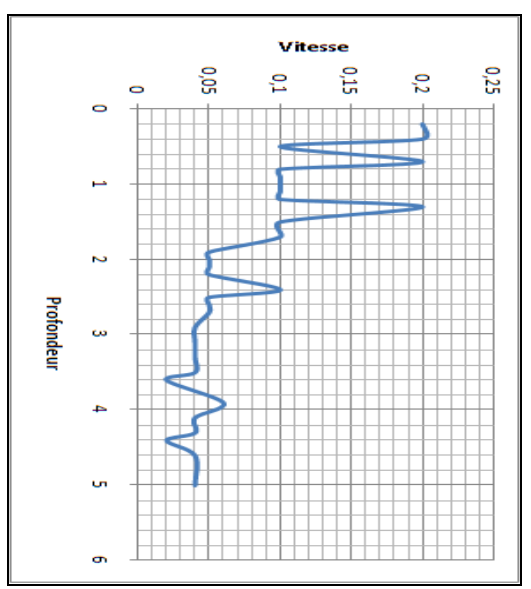

Figure 6 : vitesse $(\mathrm{cm} / \mathrm{min})$ d'infiltration au point $n^{\circ} 4$

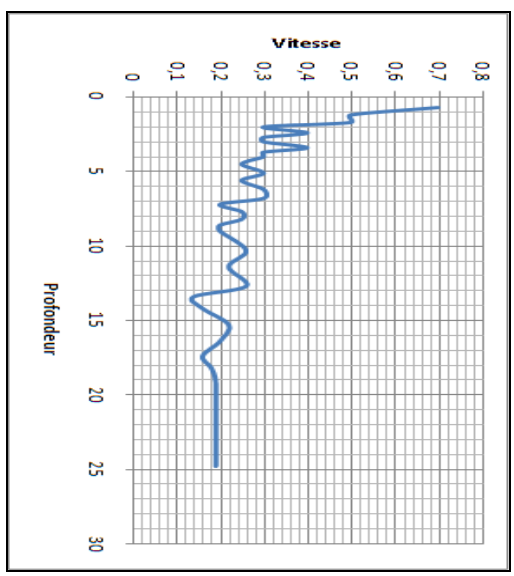

Figure 8 : vitesse $(\mathrm{cm} / \mathrm{min})$ d'infiltration au point $n^{\circ} 6$ 


\section{Mbilou et al. J. Appl. Biosci. 2016 Mesure de la vitesse d'infiltration des eaux dans le sol : Cas des sols de la Vallée du Niari en République du Congo}

\section{DISCUSSION}

Toutes les courbes d'infiltration réalisées montrent que la vitesse d'infiltration est relativement notable $(0,6$ à 0,4 $\mathrm{cm} / \mathrm{min}$ ) juste sur les 2 à 5 premiers centimètres du sol. Elle se réduit fortement en profondeur, en se stabilisant autour de $0,2 \mathrm{~cm} /$ minute jusqu'à une profondeur de 20 à $15 \mathrm{~cm}$ puis devient nulle à partir de $25-30 \mathrm{~cm}$ de profondeur. II s'agit donc d'une infiltration superficielle qui ne peut pas influencer la composition des nappes profonde. En effet, la superposition de nos profils (Fig.3) aux puits réalisés par Jean de Dieu NZILA (2013) montre bien une nette corrélation entre les différents horizons du sol et la vitesse d'infiltration. La vitesse d'infiltration est notable dans les 15-25 premiers centimètres correspondant au sol noir arable, elle décroit dans I'horizon argilo-sableux, et devient très faible dans l'horizon argileux. Cette décroissance de la vitesse d'infiltration semble être liée à l'abondance en argile dans le profil d'altération qui réduit la porosité. Ceci semble être caractéristique de tous les sols de la vallée du Niari qui se sont développés sur le schisto-calcaire.

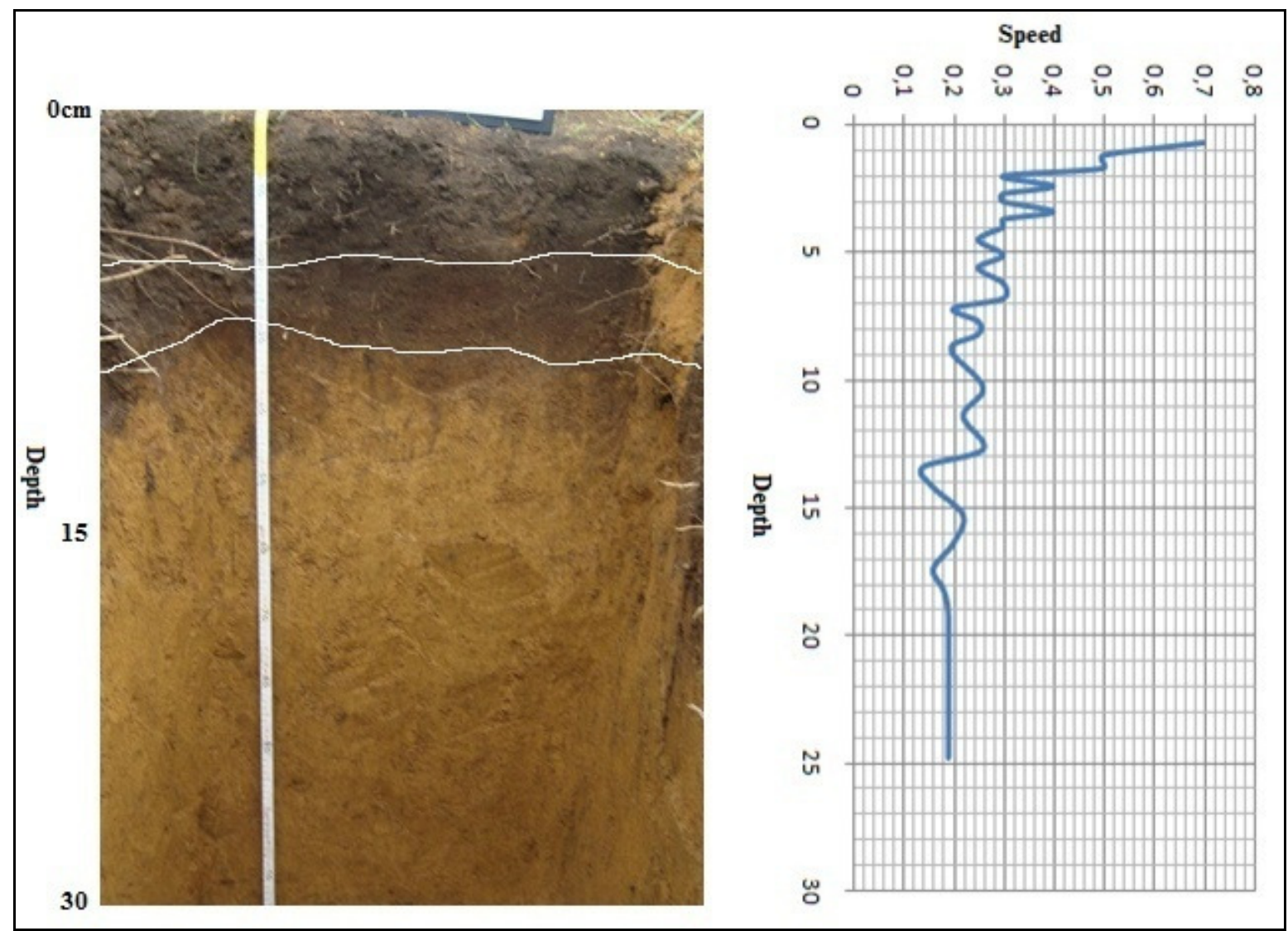

Figure 3 : Comparaison du profil des sols complexes Niari avec la vitesse d'infiltration

\section{CONCLUSION}

L'on peut donc envisager, pour la mise en valeur de ces sols une agriculture moderne, mécanisée avec irrigation éventuelle. La vallée du Niari possède de bonnes terres agricoles. Les mesures d'infiltration prouvent à suffisance qu'une agriculture mécanisée avec irrigation n'aura rien de néfaste sur les nappes d'eau souterraine et la production car les eaux n'atteindront pas si rapidement les nappes souterraines. La composition des horizons de ces sols allant du sablo-argileux (au sommet) au argileux (à la base) ralentit considérablement l'infiltration et se comporte en véritable couche protectrice protégeant ainsi les nappes d'eau souterraine des éventuelles pollutions. 


\section{REFERENCES}

Boissezon (P. de), Martin (G.), 1967 : Quinze ans de travaux de recherches dans les pays du Niari. Les sols de la vallée du Niari. Étude pédologique. P. Boiry, Monaco, $189 \mathrm{p}$.

Boulange B., 1984: Les formations bauxitiques latéritiques de Cote d'Ivoire. Les facies, leur transformation, leur distribution et l'évolution du modelé. Travaux et Doc. ORSTOM, Paris, 175, $363 \mathrm{P}$.

Bouwer, H, 1986: intake rate: Cylinder infiltrometer. In: methods of soil analysis, part I. Physical and mineralogical methods. Agronomy Monograph no.9 (2nd éd.). Am. Soc. Of Agronomy, Soil Sc. Soc. Of America.

Brugere J.M. 1965 : Étude pédologique de la vallée du Niari. ORSTOM, Paris, 2 t. 326 p. 1952-1953.

Denis B et Rieppel J.M. 1975 : Notice explicative $n^{\circ} 60$. Carte pédologique Madingou République Populaire du Congo à 1/200 000. ORSTOM, Centre Brazzaville - Paris

Green, W.H. \& G.A. Ampt, 1911: Studies in soil physics, part I. The flows of air and water through soils. J. Agric. Sci. 4.
Martin G. 1970 : Synthèse agro-pédologique des études ORSTOM dans la Vallée du Niari en République du Congo Brazzaville. Cah. ORSTOM., ser. Pédol. Vol. VIII, n॰1.

Mboukou-Kimbatsa I. M. C. 1998: Les macros invertébrés du sol dans les différents systèmes d'agriculture au Congo. Thèse de doctorat 195 p. Université Pierre et Marie Curie, Paris, France.

Munsell Color, 1990: Munsell soil color charts, Kole Morgen Instruments. Maryland.

Ngouala-Mabonzo M. 2008 : Étude hydrogéologique de la nappe du bassin supérieur de la Loémé (Mayombe). Mémoire de Maitrise en géographie physique 68 p. Université Marien Ngouabi, Brazzaville, Congo.

Nzila J.D.D., 22013 : Etat, besoins et priorités pour une gestion durable des sols du Congo-Brazzaville. Launch of global soil partnership in western and central Africa; 4 - 6 February 2013. Labadi Beach Hotel, Accra, Ghana.

Renard F. 2002: Cours d'hydrogéologie, Master professionnel DESS, $54 \mathrm{p}$. 